\title{
SPOUSAL CAREGIVING IS ASSOCIATED WITH AN INCREASED RISK OF FRAILTY: A CASE-CONTROL STUDY
}

\author{
F. POTIER ${ }^{1,2}$, J.-M. DEGRYSE ${ }^{2,3}$, G. AUBOUY ${ }^{1}$, S. HENRARD ${ }^{2,4}$, B. BIHIN ${ }^{5}$, F. DEBACQ-CHAINIAUX ${ }^{6}$, \\ H. MARTENS ${ }^{7}$, M. de SAINT-HUBERT ${ }^{1,2}$
}

\begin{abstract}
1. Department of Geriatrics, CHU Université Catholique de Louvain, Namur, Belgium; 2. Institute of Health and Society, Université Catholique de Louvain, Brussels, Belgium; 3. Departments of Public Health and Primary Care, Katholieke Universiteit Leuven, Leuven, Belgium; 4. Clinical Pharmacy Research Group, Louvain Drug Research Institute, Université catholique de Louvain, Brussels, Belgium; 5. Scientific Support Unit, CHU Université Catholique de Louvain, Namur, Belgium; 6. Unit of Research on Cellular Biology, NARILISNamur Research Institute for Life Sciences, University of Namur, Belgium; 7. GIGA Research Institute, University of Liège, Liège, Belgium. Corresponding author: Florence Potier M.D., Department of Geriatrics, Centre Hospitalier Universitaire Université Catholique de Louvain Namur, 1, rue Dr G. Therasse, 5530 Mont-
\end{abstract} Godinne, Belgium. Tel 0032/81422175.Fax: 0032/81423885. Email: florence.potier@uclouvain.be

\begin{abstract}
Background: Evidence suggests that providing care for a disabled elderly person may have implications for the caregiver's own health (decreased immunity, hypertension, and depression). Objective: Explore if older spousal caregivers are at greater risks of frailty compared to older people without a load of care. Design: Case-control study. Setting: Participants were assessed at home in Wallonia, Belgium. Participants: Cases: community-dwelling spousal caregivers of older patients, recruited mainly by the geriatric outpatient clinic. Controls: people living at home with an independent spouse at the functional and cognitive level matched for age, gender and comorbidities. Measurements: Mini nutritional assessment-short form (MNA-SF), short physical performance battery (SPPB), frailty phenotype (Fried), geriatric depression scale (GDS-15), clock drawing test, sleep quality, and medications. The multivariable analysis used a conditional logistic regression. Results: Among 79 caregivers, 42 were women; mean age and Charlson comorbidity index were $79.4 \pm 5.3$ and $4.0 \pm 1.2$, respectively. Among care-receivers (mean age $81.4 \pm 5.2$ ), $82 \%$ had cognitive impairment. Caregiving was associated with a risk of frailty (Odd Ratio (OR) 6.66; 95\% confidence interval (CI) 2.20-20.16), the consumption of antidepressants (OR 4.74; 95\% CI 1.32 -17.01), shorter nights of sleep (OR 3.53; 95\% CI 1.37-9.13) and more difficulties maintaining a social network (OR 5.25; 95\% CI 1.68-16.40). Conclusions: Spousal caregivers were at an increased risk of being frail, having shorter nights of sleep, taking antidepressants and having difficulties maintaining their social network, compared to non-caregiver controls. Older spousal caregivers deserve the full attention of professionals to prevent functional decline and anticipate a care breakdown.
\end{abstract}

Key words: Caregiving, frailty, nutrition, depression.

\section{Introduction}

In $2014,34 \%$ of the population in 20 European countries provided care to a family member (1). Among older couples, spouses are first in line to provide care (2), and they are key elements in the home support of dependent older persons (3).

As we followed our patients in geriatric consultations, it seemed to us that spouses gave care until exhaustion. In this context, we wondered how to assess the health of older spousal caregivers.

Informal care could be associated with a reduction in physical and mental health. For instance, evidence suggests that caregivers have decreased immunity observed with a lower antibody response to vaccines $(4,5)$, have higher rates of hypertension and coronary diseases $(6,7)$ and are at greater risk for depression and anxiety (8).

In later life, these effects on health may lead to increased frailty (9). The frailty phenotype is generally defined as a decline in homeostatic reserves in multiple physiological systems, resulting in greater vulnerability (10). Frailty as a phenotype has significant public health relevance because frail older adults have a greater risk of falls, disability, hospitalization, institutionalization, and death $(10,11)$. These risks could anticipate a care breakdown and therefore could result in hospitalizations or nursing home admission of the carereceiver.

To date, little is known about the risk of frailty among spousal caregivers. Therefore, the purpose of this study is to explore if older spousal caregivers are at greater risk for frailty compared to older people without a load of care.

\section{Methods}

This was a cross-sectional analysis of the baseline data from a cohort study of older spousal caregivers.

\section{Participants}

Caregivers were recruited through the geriatric outpatient clinic and the memory centre of the University Hospital of Louvain in Namur and because of the efforts of general practitioners and home nurses from March 2015 until May 2016. Cases were defined as spouses of older patients with 
a cognitive deficit (score of more than 2/7 on the Global Deterioration Scale (12)) or functional impairment (a minimum dependence of 1 activity in daily living) who were still living at home. Controls were people living at home with an independent spouse at the functional and cognitive level. Controls were recruited with the help of general practitioners and home nurses and via senior associations. Controls were matched for age, gender, and comorbidity.

All participants had to be 70 years or older. All provided written informed consent that was approved by the CHU UCL Namur Institutional Review Board (NUB: B039201422799).

\section{Socio-demographic data}

Participants were assessed at home. The following data were collected: age, gender, educational level and income level. Furthermore, medical history was taken including any medications, smoking status, alcohol consumption, sleep duration and sleep perturbation. Socioeconomic status was constructed as suggested by Cockerham (13). A total score was calculated as the sum of education (primary school=1; unfinished secondary education $=2$; secondary education $=3$; higher education=4), income (difficult=1; easy=2) and past occupation (worker-farmer-unemployed $=1$; craftsmanself-employed=2; employee-officer $=3$, manager-liberal profession=5). Questions also addressed their social network including family, friends or neighbours on a 5-point Likert-type scale $(1=$ very difficult to maintain contact; $5=$ very easy to maintain contact).

\section{Medical data}

Comorbidity was evaluated with the Charlson Comorbidity Index (14). The occurrence of falls, hospitalizations and the numbers of medications were recorded. Body mass index (BMI) was computed as the ratio between weight in kilograms and height in square metres. The short physical performance battery (15) assessing lower extremity function included balance (ability to stand in tandem positions), gait speed (time to walk 4 metres) and strength (time to rise from a chair and return to the seated position 5 times). Nutrition was assessed with the mini Nutritional Assessment short form (16); a risk of malnutrition was considered for total scores below 12/14. The frailty phenotype was assessed according to the definition of $\mathrm{L}$. Fried (10), a pre-frail status considered for a total score of 1 or 2 out of 5 and a frail status for a total score above $2 / 5$. The grip strength of the dominant hand was measured with the Martin vigorimeter. The highest score of the three trials was retained. Finally, cognitive status was evaluated with the clock drawing test (17).

\section{Psychosocial data}

The sense of coherence of the participants was measured with the Sense of Coherence Scale (SOC-13), a 7-point Likerttype scale (18). SOC seems to be a health-promoting resource that strengthens resilience and develops a positive subjective state of health. Depressive symptoms were screened with the Geriatric Depression Scale-15 (19); a participant was considered at risk of depression with a score above 5/15.

\section{Additional data of caregivers}

Caregivers completed two additional self-report questionnaires. The Caregiver Reaction Assessment (CRA) (20) was used to estimate the various aspects of the caregiving situation by considering positive dimensions such as selfesteem. Caregiver burden was measured using the Zarit Burden Interview (ZBI) (Zarit et al. 1980) that consists of 22 selfreported items. We also collected the following information concerning the caregiving situation: the time spent giving care or supervision, the duration of being a caregiver, the activities of daily living they perform and the informal and professional support they have.

\section{Medical data of care-receiver}

Functional impairment was evaluated with the Katz Index (21) on a 6-point scale, with lower scores indicating greater dependence. In cognitive disorder cases, the severity of dementia was rated with the Global Deterioration Scale (12), and behavioural disturbances were screened with the Neuropsychiatric Index $(22,23)$. All data for the care-receiver were completed by their caregivers.

\section{Statistical analyses}

The sample size (79 caregivers and 79 controls) was calculated with an expected difference between caregivers and controls in Interleukin-6 (IL-6), a pro-inflammatory biomarker. Indeed, previous observational studies have found an association between frailty and elevated levels of proinflammatory mediators, such as IL-6, implicating a chronic, pro-inflammatory state in the pathogenesis of frailty (24). The biological results will not be discussed in this article.

Variables were compared between caregivers and controls using the Mac Nemar test for categorical variables and the Wilcoxon signed rank test for continuous measures. Variables that were significantly different between caregivers and controls were entered into a conditional logistic regression. Then, a stepwise selection based on the Akaike's information criterion (AIC) was performed to select the final multivariable model. The results are presented as odd ratios (OR) and 95\% confidence intervals (CI). We did not consider age, gender and comorbidities because controls were matched on these variables. Data were analysed using the SPSS statistical software package (version 24; SPSS Inc., Chicago, IL, USA) and R statistical software Version 3.3.1. (R Foundation for Statistical Computing, Vienna, Austria). Statistical tests were two-tailed, and a P-value $<0.05$ was considered significant. 


\section{CAREGIVER'S FRAILTY}

\section{Results}

\section{Descriptive analysis}

A total of 79 community-dwelling spousal caregivers of older patients were recruited $(49 \%$ by the geriatric outpatient clinic, $11 \%$ by the memory centre, $13 \%$ by general practitioners, $9 \%$ by home nurses, and $18 \%$ through different senior associations). The median age was 79.0 years [76$84]$, and the sample was almost equivalent in gender $(53 \%$ of women). Care-receivers' median age was 81 years [78-85]. A large majority $(82 \%)$ of the care-receivers had cognitive impairment, and $68 \%$ had cognitive impairment with behavioural disorders. Their functional status was variable with a median of 3 [1-5] notes of 6 on the Katz ADL scale.

Table 1

Caregiver and control socio-demographic and psychological variables at baseline

\begin{tabular}{lccc}
\hline & $\begin{array}{c}\text { Caregivers } \\
(\mathbf{N}=79)\end{array}$ & $\begin{array}{c}\text { Controls } \\
(\mathbf{N}=79)\end{array}$ & p-value \\
\hline Gender, female, n (\%) & $42(53.2)$ & $42(53.2)$ & $>0.99$ \\
Age, years, median [P25-P75] & $79.0[76-84]$ & $78.0[75-82]$ & 0.09 \\
Socioeconomic status lowest quartile ${ }^{\mathrm{a}}, \mathrm{n}(\%)$ & $20(25.3)$ & $17(21.5)$ & 0.57 \\
Social network, median [P25-P75] & $3.0[2-4]$ & $2.0[2-3]$ & $<0.01$ \\
Risk of depression ${ }^{\mathrm{b}}, \mathrm{n}(\%)$ & $24(30.4)$ & $11(14.0)$ & 0.02 \\
Use of anti-depressive medication, n (\%) & $18(22.8)$ & $5(6.3)$ & $<0.01$ \\
Use of anxiolytic medication, n (\%) & $22(27.8)$ & $13(16.5)$ & 0.11 \\
Sense of Coherence, median [P25-P75] & $65.0[56-73]$ & $69.0[62-77]$ & 0.02 \\
\hline $\begin{array}{l}\text { a. Socioeconomic status score was calculated as the sum of education, income and past } \\
\text { occupation; b. Risk of depression: GDS>5/15 }\end{array}$ & &
\end{tabular}

\section{Univariate analysis}

The comparison of caregivers and controls on sociodemographic and psychological variables is shown in Table 1 and on clinical variables on Table 2 . We found nine variables that were significantly different between caregivers and controls. It was more difficult for caregivers to maintain their social network (family, friends, and neighbourhood). They had lower scores in physical performance and a higher risk of malnutrition and frailty (Table 2). Specifically, a higher number of caregivers had lost more than three kilograms in three months. Thirty percent of the caregivers were at risk for depression, and the consumption of antidepressants was also higher in caregivers than in controls. Caregivers reported more perturbed sleep and shorter nights ( $<8$ hours) than controls. Finally, caregivers showed lower scores in sense of coherence.

\section{Multivariable analysis}

The results of the conditional logistic regression are shown in Table 3. Our model showed that for the same age, gender and comorbidities, caregiving is associated with a risk of frailty, the consumption of antidepressants, shorter nights of sleep and more difficulties maintaining a social network.

\section{Table 2}

Caregiver and control clinical variables at baseline

\begin{tabular}{lccc}
\hline & $\begin{array}{c}\text { Caregivers } \\
(\mathbf{N}=79)\end{array}$ & $\begin{array}{c}\text { Controls } \\
(\mathbf{N = 7 9 )}\end{array}$ & p-value \\
\hline Charlson comorbidity index, Median [P25-P75] & $4[3-5]$ & $4[3-5]$ & 0.76 \\
Ever smoker, n (\%) & $11(13.9)$ & $9(11.4)$ & 0.62 \\
Hospitalization < 1 year, n (\%) & $14(17.7)$ & $16(20.3)$ & 0.68 \\
Fall previous year, n (\%) & $21(26.6)$ & $24(30.4)$ & 0.60 \\
$>2$ glasses alcohol/day, n (\%) & $17(21.5)$ & $19(24.1)$ & 0.68 \\
Number of medications, median [P25-P75] & $3.0[2-5]$ & $4.0[2-5]$ & 0.78 \\
SPPB ${ }^{a}$, median [P25-P75] & $9.0[6-10]$ & $10[8-11]$ & $<0.01$ \\
Frailty (Fried), median [P25-P75] & $2.0[1-2]$ & $1.0[1-2]$ & $<0.01$ \\
Grip strength, median [P25-P75] & $70[57-78]$ & $68[56-79]$ & 0.53 \\
BMI, median [P25-P75] & $26[24-28]$ & $27[24-30]$ & 0.11 \\
Nutrition (MNA), median [P25-P75] & $12[10-14]$ & $13[12-14]$ & $<0.01$ \\
Cognition ${ }^{b}, n(\%)$ & $35(44.3)$ & $30(38.0)$ & 0.41 \\
Perturbed sleep, n (\%) & $31(39.2)$ & $14(17.7)$ & $<0.01$ \\
Sleep < 8 h/night, n (\%) & $50(63.3)$ & $30(38.0)$ & $<0.01$ \\
\hline
\end{tabular}

a. SPPB: Short Physical Performance Battery (score 0-12); b. Cognition: pathologic clock drawing test

\section{Frailty}

Exploratory analysis was made to identify the factors associated with caregivers' frailty (Table 4). We could not find

Table 3

Conditional logistic regression comparing caregivers and controls

\begin{tabular}{|c|c|c|c|c|}
\hline \multirow[b]{2}{*}{ Variable } & \multicolumn{2}{|c|}{ Univariate analysis } & \multicolumn{2}{|c|}{ Multivariable analysis } \\
\hline & OR $(95 \% \mathrm{CI})$ & p-value & OR $(95 \% \mathrm{CI})$ & p-value \\
\hline Social network ${ }^{\text {a }}$ & $5.90(2.03-11.87)$ & $<0.01$ & $5.25(1.68-16.40)$ & $<0.01$ \\
\hline Frail-prefrail & $5.35(2.38-12.05)$ & $<0.01$ & $6.66(2.20-20.16)$ & $<0.01$ \\
\hline Short nights ( $<8$ hours $)$ & $2.60(1.34-5.05)$ & $<0.01$ & $3.53(1.37-9.13)$ & $<0.01$ \\
\hline Use of anti-depressive medication & $3.53(1.34-9.35)$ & 0.01 & $4.74(1.32-17.01)$ & 0.02 \\
\hline Low-intermediate physical performance & $2.24(1.16-4.32)$ & 0.02 & $1.08(0.40-2.90)$ & 0.88 \\
\hline
\end{tabular}

a. Social network: difficult or very difficult to maintain the social network 
THE JOURNAL OF FRAILTY \& AGING

Table 4

Factors associated with caregiver frailty in logistic regression

\begin{tabular}{|c|c|c|c|c|}
\hline \multirow[b]{2}{*}{ Variable } & \multicolumn{2}{|c|}{ Univariate analysis } & \multicolumn{2}{|c|}{ Multivariable analysis } \\
\hline & OR $(95 \% \mathrm{CI})$ & p-value & OR $(95 \% \mathrm{CI})$ & p-value \\
\hline Caregiver's age & $.09(0.99-1.19)$ & 0.08 & $1.02(0.92-1.14)$ & 0.68 \\
\hline Caregiver's Charlson & $1.13(0.69-1.86)$ & 0.62 & & \\
\hline Caregiver's self-esteem & $1.04(0.56-1.93)$ & 0.90 & & \\
\hline Caregiver's burden & $0.97(0.94-1.01)$ & 0.10 & $0.97(0.93-1.00)$ & 0.07 \\
\hline Patient's functionality ${ }^{a}$ & $0.91(0.73-1.13)$ & 0.39 & & \\
\hline Patient's cognition ${ }^{\mathrm{b}}$ & $0.95(0.69-1.31)$ & 0.75 & & \\
\hline Caregiving's respite & $0.34(0.11-1.08)$ & 0.07 & $0.37(0.10-1.44)$ & 0.15 \\
\hline Home nurses support & $3.1(1.17-8.23)$ & 0.02 & $5.80(1.71-19.64)$ & $<0.01$ \\
\hline Caregiving $>3$ years & $0.28(0.08-0.92)$ & 0.04 & $0.20(0.05-0.79)$ & 0.02 \\
\hline$>2 \mathrm{~h}$ of care/day & $1.05(0.41-2.70)$ & 0.93 & & \\
\hline
\end{tabular}

a. Katz Index on a 6-point scale; b. Global Deterioration Scale

a relationship between frailty and age, comorbidity, burden or self-esteem of the caregiver. Caregivers' frailty was not associated with cognitive or functional status of the carereceiver. However, frailty was associated with the involvement of a nurse at home (OR 5.80; 95\%CI 1.71-19.64). Caregivers involved in care for more than three years were at a lower risk of frailty.

\section{Discussion}

Our study identified that older spousal caregivers were more likely to present with frailty, perturbed sleep, difficulties maintaining a social network and use of anti-depressive drugs than people without this load of care. After the multivariable analysis, caregivers showed a six times greater risk of being frail compared with non-caregiver controls. We screened frailty with the definition of L. Fried and focused on physical indicators including muscle strength, endurance or weight loss. More precisely, caregivers were more likely to be in a pre-frail stage (one or two present criteria), which is identified as a high risk of progressing to frailty (10). The difference was especially shown in the criteria of unintentional weight loss (more than $4.5 \mathrm{~kg}$ within the past year) and low physical activity. It is important to note that these two criteria are also considered in the MNA score. According to MNA, 35\% of the caregivers were at risk for malnutrition. No differences were observed between male and female caregivers in nutritional status, although Puranen et al. (25) found that male gender caregivers were associated with a lower nutrient intake.

We found that caregiver frailty was associated with the attendance of a nurse at home. Home nurses are, thus, potentially well placed to detect frailty of the spouses of their patients. In contrast, giving care for more than three years was associated with more caregiver robustness. This is consistent with the study of Fredman et al., which notes that it is necessary to be healthy to remain a caregiver (26).

Caregivers reported more sleep problems than controls (perturbed sleep and shorter nights). Caregivers' sleep deficits have already been confirmed with objective sleep assessments such as polysomnography $(27,28)$.

Finally, older spousal caregivers were more likely to use anti-depressive drugs. Several reviews indicated a positive association between frailty and depression (29, 30). Actually, depression and frailty share presenting symptoms, such as low daily activity profiles, that could result from exhaustion and loss of interest.

The theoretical framework underlying the assertion that caregiving is associated with a reduction in physical health is based on a model of the impact of stress on health (31). Caregivers may experience distress when their resources become insufficient (information, coping, finances, lack of respite, etc.). However, in our study, positive experiences, such as self-esteem, or on the contrary, negative experiences, such as the burden, were not associated with caregiver frailty.

The risk of frailty associated with caregiving can also be partly explained by the fact that caregivers are less likely to engage in preventive health behaviours. Indeed, in the Caregiver Health Effects Study, having a spouse with an ADL impairment predicted poor preventive health behaviours on the part of the caregiver, including not finding time for exercise, inadequate rest and forgetting to take medications (32).

A potential confounding factor that could explain the differences in health outcomes between caregivers and controls could be "assortative mating". Assortative mating means that people select spouses that have similar lifestyle factors that may influence their risk of negative health outcome (33).

A recent paper based on the Health and Retirement Study showed that dementia caregivers were significantly more likely to experience increased frailty relative to non-dementia caregivers (34). To calculate this outcome, they developed a 
frailty index based on available data from a survey: chronic illnesses, ADL and IADL limitations, depression, obesity and self-rated health.

To our knowledge, our study is the first assessing the health of old spousal caregivers based on clinical data collected at participants' homes. Furthermore, the majority of the studies assessing caregivers concern female caregivers. Our sample was almost equivalent in gender.

\section{Limitations}

This study has limitations that should be considered when interpreting the results. First, because these are cross-sectional data, we cannot establish causality between caregiving and negative health outcomes. Second, both groups are convenience samples, and the recruitment methods were different between the caregiving group (more recruitment from the geriatric outpatient clinic) and the control group (more recruitment from general practitioners and senior associations). Third, the control group was matched for gender, age and comorbidities with Charlson's index, which might not be the most precise tool to assess the comorbidity of geriatric patients. Furthermore, all data were completed by the caregivers. Lastly, this study concerns a specific caregiving subtype; spousal caregivers of geriatric patients that most suffer from cognitive deficit, thus limiting the generalizability of our results.

\section{Implications}

Healthcare providers have a role to play in the prevention of caregiver frailty, e.g., to develop advice relative to nutrition and depression screening. They should also propose solutions for home-care, allowing caregivers to share a minimum of social activities and find time for exercise.

In conclusion, many caregivers of geriatric patients are spouses who are old themselves. These spousal caregivers are at an increased risk of being frail, having shorter nights of sleep, taking antidepressants and having difficulties maintaining their social network. Older spousal caregivers deserve the full attention of professionals to prevent functional decline and anticipate a care breakdown

Acknowledgments: We would like to thank Dr. Eric Mormont from the memory centre of the University Hospital of Louvain in Namur, Aide et Soins à Domicile Namur and the general practitioners for referring participants to the project.

Funding: This work was supported by the Walloon region, Fond d'innovation sociale "Germaine Tillion" convention 1318184. The sponsors had no role in the design and conduct of the study, in the collection, analysis, and interpretation of data; in the preparation of the manuscript; or in the review or approval of the manuscript.

Disclosure of interest: The authors report no conflicts of interest.

\section{References}

1. Verbakel E, Tamlagsronning S, Winstone L, Fjaer EL, Eikemo TA. Informal care in Europe: findings from the European Social Survey (2014) special module on the social determinants of health. Eur J Public Health 2017;27(suppl 1):90-95.

2. Deboosere P, Demarest S, Lorant V, Miermans P-J, Portet M-I, Oyen HV. Santé et soins informels. 2001.

3. Yaffe K, Fox P, Newcomer R, Sands L, Lindquist K, Dane K, et al. Patient and caregiver characteristics and nursing home placement in patients with dementia. Jama 2002;287(16):2090-7.

4. Glaser R, Sheridan J, Malarkey WB, MacCallum RC, Kiecolt-Glaser JK. Chronic stress modulates the immune response to a pneumococcal pneumonia vaccine. Psychosom Med 2000;62(6):804-7.

5. Li J, Cowden LG, King JD, Briles DA, Schroeder HW, Jr., Stevens AB, et al. Effects of chronic stress and interleukin-10 gene polymorphisms on antibody response to tetanus vaccine in family caregivers of patients with Alzheimer's disease. Psychosom Med 2007;69(6):551-9.

6. Shaw WS, Patterson TL, Ziegler MG, Dimsdale JE, Semple SJ, Grant I. Accelerated risk of hypertensive blood pressure recordings among Alzheimer caregivers. J Psychosom Res 1999;46(3):215-27.

7. Capistrant BD, Moon JR, Berkman LF, Glymour MM. Current and long-term spousa caregiving and onset of cardiovascular disease. J Epidemiol Community Health 2012;66(10):951-6.

8. Pinquart M, Sorensen S. Differences between caregivers and noncaregivers in psychological health and physical health: a meta-analysis. Psychol Aging 2003;18(2):250-67.

9. Chang SS, Weiss CO, Xue QL, Fried LP. Association between inflammatory-related disease burden and frailty: results from the Women's Health and Aging Studies (WHAS) I and II. Arch Gerontol Geriatr 2012;54(1):9-15.

10. Fried LP, Tangen CM, Walston J, Newman AB, Hirsch C, Gottdiener J, et al. Frailty in older adults: evidence for a phenotype. J Gerontol A Biol Sci Med Sci 2001;56(3):M146-56

11. Bandeen-Roche K, Xue QL, Ferrucci L, Walston J, Guralnik JM, Chaves P, et al. Phenotype of frailty: characterization in the women's health and aging studies. J Gerontol A Biol Sci Med Sci 2006;61(3):262-6.

12. Reisberg B FS, de Leon MJ, Crook T. The Global Deterioration Scale for assessment of primary degenerative dementia. American Journal of Psychiatry 1982;139(9):1136-1139.

13. Cockerham WC. Health lifestyles and the absence of the Russian middle class. Sociol Health Illn 2007;29(3):457-73.

14. Charlson ME, Pompei P, Ales KL, MacKenzie CR. A new method of classifying prognostic comorbidity in longitudinal studies: development and validation. J Chronic Dis 1987:40(5):373-83

15. Guralnik JM, Simonsick EM, Ferrucci L, Glynn RJ, Berkman LF, Blazer DG, et al A short physical performance battery assessing lower extremity function: association with self-reported disability and prediction of mortality and nursing home admission. J Gerontol 1994:49(2):M85-94.

16. Kaiser MJ, Bauer JM, Ramsch C, Uter W, Guigoz Y, Cederholm T, et al. Validation of the Mini Nutritional Assessment short-form (MNA-SF): a practical tool for identification of nutritional status. J Nutr Health Aging 2009;13(9):782-8.

17. Kokmen E, Smith GE, Petersen RC, Tangalos E, Ivnik RC. The short test of mental status. Correlations with standardized psychometric testing. Arch Neurol 1991;48(7):725-8

18. Eriksson M, Lindstrom B. Validity of Antonovsky's sense of coherence scale: a systematic review. J Epidemiol Community Health 2005;59(6):460-6.

19. Lesher EL, Berryhill JS. Validation of the Geriatric Depression Scale--Short Form among inpatients. J Clin Psychol 1994;50(2):256-60.

20. Pascal Antoine SQ, Véronique Christophe. Valeurs psychométriques CRA Vivre avec un proche malade :évaluation des dimensions positive et négative de l'expérience des aidants naturels. 2007.

21. Katz S. Assessing self-maintenance: activities of daily living, mobility, and instrumental activities of daily living. J Am Geriatr Soc 1983;31(12):721-7.

22. Cummings JL, Mega M, Gray K, Rosenberg-Thompson S, Carusi DA, Gornbein J. The Neuropsychiatric Inventory: comprehensive assessment of psychopathology in dementia. Neurology 1994;44(12):2308-14.

23. Kaufer DI, Cummings JL, Ketchel P, Smith V, MacMillan A, Shelley T, et al. Validation of the NPI-Q, a brief clinical form of the Neuropsychiatric Inventory. J Neuropsychiatry Clin Neurosci 2000;12(2):233-9.

24. Leng SX, Xue QL, Tian J, Walston JD, Fried LP. Inflammation and frailty in older women. J Am Geriatr Soc 2007;55(6):864-71.

25. Puranen TM, Pietila SE, Pitkala KH, Kautiainen H, Raivio M, Eloniemi-Sulkava U, et al. Caregivers' male gender is associated with poor nutrient intake in AD families (NuAD-trial). J Nutr Health Aging 2014;18(7):672-6.

26. Fredman L, Lyons JG, Cauley JA, Hochberg M, Applebaum KM. The Relationship Between Caregiving and Mortality After Accounting for Time-Varying Caregiver Status and Addressing the Healthy Caregiver Hypothesis. J Gerontol A Biol Sci Med Sci 2015. 


\section{THE JOURNAL OF FRAILTY \& AGING}

27. Fonareva I, Amen AM, Zajdel DP, Ellingson RM, Oken BS. Assessing sleep architecture in dementia caregivers at home using an ambulatory polysomnographic system. Journal of Geriatric Psychiatry and Neurology 2011;24(1):50-59.

28. Von Känel R, Ancoli-Israel S, Dimsdale JE, Mills PJ, Mausbach BT, Ziegler MG, et al. Sleep and biomarkers of atherosclerosis in elderly Alzheimer caregivers an controls. Gerontology 2010;56(1):41-50.

29. Buigues C, Padilla-Sanchez C, Garrido JF, Navarro-Martinez R, Ruiz-Ros V, Cauli $\mathrm{O}$. The relationship between depression and frailty syndrome: a systematic review. Aging Ment Health 2015;19(9):762-72.

30. Mezuk B, Edwards L, Lohman M, Choi M, Lapane K. Depression and frailty in later life: a synthetic review. Int J Geriatr Psychiatry 2012;27(9):879-92.
31. McEwen BS. Protective and damaging effects of stress mediators. N Engl J Med 1998;338(3):171-9.

32. Burton L. C. JTN, R Schulz, C. H. Hirsch, P.S. German. Preventive Health Behaviors among Spousal Caregivers. 1997.

33. Buss DM. Toward a psychology of person-environment (PE) correlation: The role of spouse selection. Journal of Personality and Social Psychology 1984;47(2):361-377.

34. Dassel KB, Carr DC. Does Dementia Caregiving Accelerate Frailty? Findings From the Health and Retirement Study. Gerontologist 2016;56(3):444-50. 